\section{EDUCATION}

Research, Innovation and Solutions on-line ${ }^{\circledR}$
Electronic Journal of Research

in Educational Psychology

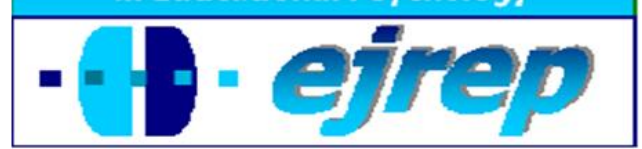

\title{
Perfiles cognitivos en alumnos con baja, media y alta creatividad
}

\author{
Mercedes Ferrando, Rosario Bermejo, Marta Sainz, \\ Carmen Ferrándiz, María Dolores Prieto, Gloria Soto
}

Facultad de Educación, Universidad de Murcia

\section{España}

Correspondencia: Mercedes Ferrando Prieto. Universidad de Murcia, Facultad de Educación, Campus de Espinardo 30100. Murcia (España).Teléfono: +3486888 8196. E-mail: mferran@um.es

(C) Education \& Psychology I+D+i and Editorial EOS (Spain) 


\section{Resumen}

Introducción. El objetivo de este trabajo es avanzar en el estudio de la relación entre creatividad e inteligencia, analizando los perfiles cognitivos de los alumnos. Se formula la hipótesis siguiente: si la creatividad se produce como resultado de unir conceptos distantes, un perfil más equilibrado entre las habilidades cognitivas, -un perfil plano- podría facilitar una relación débil entre conceptos, por lo tanto, los individuos creativos podrían presentar un perfil cognitivo con menos picos y valles.

Método. En este studio han participado 679 alumnos de Educación Secundaria Obligatoria, de edades comprendidas entre $\operatorname{los} 12$ y $\operatorname{los} 17$ años $(\mathrm{M}=13.86$; DT $=1.25)$. Los instrumentos utilizados fueron el TTCT y el DAT-5. Los alumnos fueron divididos en tres grupos según el nivel de creatividad: bajo, medio y alto. El perfil cognitivo de cada grupo se analizó en busca de picos.

Resultados. Los resultados indican que, contrariamente a nuestra hipótesis, el perfil cognitivo de los alumnos creativos no es diferente al de sus compañeros no creativos.

Discusión y Conclusiones. Cabe la posibilidad de que la hipótesis del perfil plano de los alumnos creativos no se confirme en una población normal, ya que los alumnos poco inteligentes tieneden a mostrar poca creatividad. La hipotesis sería útil para explicar las diferencias en creatividad entre alumnos de medio-alto nivel intellectual.

Palabras Clave: Creatividad, análisis de perfiles cognitivos, inteligencia. Teoría del umbral. 


\begin{abstract}
Introduction. The aim of the present work is to advance in the study of creativity-intelligence relationship looking into the students' cognitive profiles. It is hypothesised that, if creativity occurs as a result of relating distant concepts, a more balanced profile among cognitive abilities - a flat profile - could facilitate a loose relationship between concepts. Therefore, creative individuals would present a cognitive profile with less peaks and troughs.

Method. 679 secondary students, aged between 12 and 17 years old $(\mathrm{M}=13.86, \mathrm{SD}=1.25)$ took part in this study. The TTCT and the DAT-5 were used. Students were divided into three groups: those with low, average and high creativity. The cognitive profile of each group was analyzed searching for peaks.

Results. Results point out that, contrary to our hypothesis, the cognitive profile of creative individuals is not different from their non-creative peers.

Discussion and Conclusion. It is possible that the hypothesis of a flatter cognitive profile of highly creative students does not work for a normal population, since students with lower intelligence also tend to show lower creativity. This hypothesis may be useful to explain diferences in creativity in students with medium-high intelligence.
\end{abstract}

Keywords: Creativity, cognitive profile analysis, intelligence, threshold theory.

Received: 10/15/12 Initial acceptance: 10/20/12 Final acceptance: 11/19/12 


\section{Introducción}

El presente trabajo tiene como objetivo contribuir al estudio de la relación entre la inteligencia y la creatividad. La relación entre estos dos conceptos ha sido estudiada en términos de correlaciones entre ellos (e.g. Ferrando, Prieto, Ferrándiz, Sánchez, 2005; Getzels y Jackson, 1962; Hocevar, 1980; Kim, 2006; Mednick y Andrews, 1967; Sen y Hagtvet, 1993), según la relación de predicción de uno sobre el otro (e.g. Batey, Furnham y Safiullinab, 2010; Furnham y Bachtiar, 2008; Schubert, 1973; Silvia, 2008; Silvia y Beaty, 2012) y en función de las diferencias individuales entre los alumnos de baja versus alta creatividad o inteligencia (e.g. Getzels y Jackson, 1962; Preckel, Holling y Wiese, 2006; Runco y Albert, 1986). Llendo más allá, Nushaum y Silvia (2011) han propuesto una nueva forma de ver el problema de la relación entre la creatividad y la inteligencia mediante el uso de modelos de ecuaciones estructurale. Los resultados de sus estudios apuntan que las diferencias individuales en inteligencia fluida (Gf) predicen significativamente la creatividad, y este resultado fue parcialmente explicado por los efectos de la inteligencia fluida en el cambio ejecutivo (número de categorías diferentes que se utilizan, por ejemplo flexibilidad) durante la tarea.

Los resultados de los estudios empiricos sobre la relación entre creatividad e inteligencia han conducido a los investigadores a especular sobre cinco posibles formas en las que ambos constructos se relacionan: desde que sean constructos sin ninguna relación entre ellos, hasta que sean el mismo constructo (para realizar una revisión consultar Batey y Furnham, 2006; Kim, Cramond y VanTassel-Baska, 2010; Sternberg y O`Hara, 1999). Una de las cinco formas en las que ambos constructos podrían estar relacionados es sobreponiendose. Según esta perspectiva la relación entre ambos es más débil para puntuaciones altas de CI. Esto se conoce como la teoría del umbral (Guilford y Hoepfner, 1971; Torrance, 1962). Esta teoría afirma que los estudiantes que son creativos tienen un nivel mínimo de inteligencia, mientras que los alumnos inteligentes no siempre son los creativos. Estudios recientes no han podido comprobar esta teoría (Ferrando, Prieto, Ferrandiz y Sánchez, 2005; Kim, 2005; Naderi y Abdullah, 2010; Preckel, Holling y Wiese, 2006; Runco y Albert, 1986). Como mantienen Nushaum y Silvia "según el punto de vista de la investigación contemporánea, la creatividad y la inteligencia son rasgos distintivos que están sólo modestamente relacionados" (2011, p. 36-37). Sin embargo, aún conociendo el rango específico de inteligencia para que la relación sea más fuerte o hasta qué grado la inteligencia predicirá la creatividad, esto nos dice muy 
poco acerca de lo que define y caracteriza a los estudiantes creativos. La investigación parece haberse estancado en este debate y están siendo estudiadas pocas alternativas.

El presente estudio da un paso adelante y cambia el enfoque en el estudio de la relación entre inteligencia y creatividad, proponiendo una nueva pregunta: ¿Cómo se diferencia el perfil cognitivo de los alumnos creativos frente a los no creativos? Este estudio no se centra en conocer si los estudiantes creativos muestran una inteligencia superior o inferior. El objetivo de este estudio es analizar los perfiles de los alumnos en términos de picos y valles; es decir, en términos de puntos fuertes y débiles. Se especula que los estudiantes de alto nivel de creatividad versus bajos, no difieren sólo en el nivel de sus capacidades cognitivas, sino más bien en la forma en que estas capacidades están configuradas en sus perfiles cognitivos. Así, el investigador no debe buscar diferencias en cuanto a la cantidad de inteligencia, sino con respecto a los patrones de sus perfiles cognitivos. La hipótesis de este trabajo es que las personas más creativas presentan un perfil cognitivo plano, el cual esta equilibrado y se caracteriza por la ausencia de picos y valles (perfil irregular).

¿Por qué observar el perfil cognitivo de los estudiantes?

Hay dos razones para empezar a analizar los perfiles cognitivos más que centrarnos en la puntuaciones individuales de cada habilidad. Por un lado, desde hace dos décadas, el psicólogo educativo y cognitivo admite que la inteligencia tiene muchas facetas, por lo que no se limita a un CI y, por tanto, no puede ser adecuadamente representada por el factor "g" (Gardner 1983; 1993; Sternberg, 1985). Aunque estas teorías están siendo comúnmente aceptadas se sigue buscando una relación entre el factor general de inteligencia y su correlación con la creatividad.

Por otro lado, es importante considerar la teoría de Mendick (1962) sobre la generación de ideas creativas, la cual establece que las ideas originales provienen de asociaciones inusuales que son facilitadas por las "jerarquías asociativas planas". La teoría de Mendick ha sido probada en términos de estructura de conocimiento (Hunter y Ligon, 2008; Runco, 2007; Weisberg, 2006), pero no en términos de estructura de habilidades. 
En este trabajo, se mantiene que tener un nivel de habilidad similar en diferentes áreas podría facilitar el movimiento a través de ellas, por lo tanto la transferencia de conocimientos de una a otra. Un nivel similar de capacidad en las diferentes áreas aseguraría que estas áreas funcionen juntas en la resolución de una tarea, en lugar de trabajar por separado según los puntos fuertes del estudiante (Castello y Batlle, 1998). Por lo tanto, los individuos creativos se caracterizan por tener un perfil más equilibrado entre las distintas capacidades cognitivas, lo que significa un perfil incluso plano. Por el contrario, los alumnos no creativos mostrarán un perfil cognitivo caracterizado por diferencias significativas entre sus fortalezas y debilidades, es decir, un perfil con picos y valles.

Aunque nuestra pregunta de investigación parece simple, no se han encontrado estudios previos que hayan utilizado este enfoque, a excepción de una Tesis de Máster no publicada (Mercader, 2012); en dicho, estudio la hipótesis del perfil plano de los alumnos creativos se confirmó parcialmente.

\section{Objetivos e hipótesis}

El objetivo de este trabajo es estudiar la relación entre la creatividad y la inteligencia, en concreto, cómo las capacidades relacionadas con la inteligencia se configuran en el perfil del individuo creativo versus no creativo. Nuestro objetivo no es estudiar su nivel de inteligencia, sino los desniveles (valles y picos) de sus perfiles cognitivos. Las hipótesis son:

a) Los alumnos con alta versus baja creatividad diferiran no solo en el nivel de sus habilidades cognitivas, sino también en la forma en que estas habilidades se configuran en su perfil cognitivo.

b) Las personas más creativas presentarán un perfil cognitivo plano, el cual está equilibrado y se caracteriza por la ausencia de picos y valles.

\section{Método}

\section{Participantes}

La muestra total estuvo compuesta por 679 alumnos de Educación Secundaria Obligatoria, con edades comprendidas entre los 12 y los 17 años $(M=13,86$; DT =1,25). De 
ellos, 316 eran varones. Los participantes asistían a diferentes centros educativos de la Región de Murcia y Alicante (España). Según el baremo publicado del DAT-5, las puntuaciones de los percentiles de los participantes oscilaron desde 44,16 en razonamiento espacial a 59,75 en velocidad y precisión perceptiva, por lo que nuestra muestra de participantes se encuentra dentro de las puntuaciones medias de la población normal.

\section{Instrumentos}

Para evaluar la creatividad se utilizó la subprueba "líneas paralelas" del Test de Pensamiento Creativo de Torrance (TTCT, Torrance, 1974), en su versión adaptada para el contexto español por Prieto, López, Ferrándiz y Bermejo (2003). En esta tarea se les pide a los estudiantes hacer tantos dibujos diferentes como puedan utilizando 30 pares de líneas paralelas. Ésta es una prueba figurativa que tiene por objetivo valorar los cuatro componentes principales de la creatividad: fluidez (la cantidad de ideas dadas), flexibilidad (ideas diferentes dadas por el estudiante); originalidad (la singularidad de sus respuestas) y elaboración (la cantidad de detalles para embellecer las ideas). La prueba permite la obtención de una puntuación de creatividad total sumando las puntuaciones de los cuatro componentes. La subprueba debe ser corregida al menos por 2 evaluadores diferentes, en total 10 evaluadores diferentes participaron en la correción de la prueba. El índice de fiabilidad de esta prueba se obtuvo con el alfa de Cronbach y el coeficiente de acuerdo entre jueces (coeficiente de correlación intraclase). La dimensión más fiable fue fluidez ( $\alpha=1$ a $\alpha=, 82 ; \mathrm{CCI}=1$ a CCI = ,70), y la menos fiable fue elaboración $(\alpha=, 92$ a $\alpha=, 67$;CI $=, 85$ to $\mathrm{CCI}=, 47)$. Resultados similares se encontraron en otros estudios (Almeida, Prieto, Ferrando, Oliveira y Ferrándiz, 2007; Ferrando, Ferrándiz, Bermejo, Parra, Sánchez y Prieto, 2007; Ferrando, Ferrándiz, Prieto, Bermejo y Sáinz, 2008).

Para evaluar el perfil cognitivo de los estudiantes se utilizó el Test de Aptitudes Diferenciales (DAT-5, Bennett, Seashore y Wesman, 2000). Esta batería se compone de siete subpruebas: razonamiento verbal, cálculo, razonamiento abstracto, relaciones espaciales, razonamiento mecánico, velocidad y precisión perceptiva, así como ortografía y lenguaje. Las cinco primeras evalúan aspectos razonamiento, mientras que las dos últimas evalúan aspectos perceptuales y habilidades motoras. La fiabilidad reportada en la versión española de este test, publicada por ediciones TEA, fue desde ,75 a ,92, dependiendo de la subprueba y el rango concreto de edad. 
Además, en el manual se reporta una consistencia interna adecuada, con correlaciones de bajas a moderadas entre las escalas, siendo más altas solamente entre razonamiento verbal y aptitud académica, y entre aptitud académica y razonamiento numérico (Bennett, Seashore y Wesman, 2000).

\section{Procedimiento}

Los profesores y los padres fueron informados de los objetivos de la investigación. La participación en este estudio fue voluntaria. Y las pruebas fueron administradas durante el horario escolar, siguiendo las instrucciones de los autores. Las pruebas fueron administradas en dos sesiones en días diferentes para evitar el cansancio de los alumnos. La primera sesión comenzó temprano por la mañana. Los estudiantes completaron la primera mitad del DAT-5, tomaron un pequeño descanso y realizaron el test de líneas paralelas. Durante la segunda sesión los alumnos terminaron de completar el DAT-5

\section{Análisis de datos}

Con el fin de facilitar el análisis de datos, las puntuaciones de las variables medidas por el DAT-5 fueron transformadas en la misma escala, por lo tanto todas las variables tenían como máximo $100^{1}$.

Los estudiantes fueron agrupados dependiendo de su nivel de creatividad siguiendo el procedimiento de Almeida y Freire (2003). Mediante este procedimiento se formaron tres grupos: estudiantes con baja creatividad (aquellos que puntuaron por debajo del percentil 25), alumnos con creatividad media (aquellos que puntuaron entre percentil 25 y 75) y alumnos con alta creatividad (aquellos que puntuaron por encima del percentil 75). Los resultados de los alumnos en el área de la creatividad variaron de 0 a 144 con una media de 64,76 y una desviación típica de 29,61. El percentil 25 fue establecido con una puntuación de 45,87 y el percentil 75 con una puntuación de 85,50.

Acontinuación, el perfil cognitivo de cada grupo se analizó en busca de picos utilizando un test de medidas repetidas. 


\section{Resultados}

\section{Estadísticos descriptivos}

En la tabla 1 y en el gráfico 1 se muestran la puntuación media obtenida por cada grupo de alumnos. En los tres grupos de alumnos la puntuación más alta fue en razonamiento mecánico y la más baja en memoria. Los estudiantes con un nivel de creatividad medio obtuvieron puntuaciones más altas que sus compañeros en casi todas las varibales, pero en precisión y en velocidad perceptiva los alumnos más creativos obtuvieron puntuaciones superiores que sus compañeros. Los alumnos con bajo nivel de creatividad puntuaron más bajo en todas las variables que sus pares, excepto en ortografía, donde los estudiantes más creativos obtuvieron la puntuación más baja.

El gráfico de las puntuaciones medias no muestra diferentes patrones de las habilidades cognitivas según el nivel de creatividad, aunque las desviaciones típicas indican que el grupo de alumnos más creativos muestra una mayor variabilidad en sus resultados.

Tabla 1: Estadísticos descriptivos para las puntuaciones en las habilidades cognitivas en los tres grupos (bajo, medio y alto nivel de creatividad)

\begin{tabular}{|c|c|c|c|c|c|c|c|c|}
\hline & \multicolumn{2}{|c|}{$\begin{array}{l}\text { Creatividad } \\
\text { Baja }(n=152)\end{array}$} & \multicolumn{2}{|c|}{$\begin{array}{c}\text { Creatividad } \\
\text { media }(n=295)\end{array}$} & \multicolumn{2}{|c|}{$\begin{array}{l}\text { Creatividad Alta } \\
\qquad(\mathrm{n}=145)\end{array}$} & \multicolumn{2}{|c|}{$\begin{array}{c}\text { MUESTRA } \\
\text { TOTAL } \\
(\mathrm{N}=679)\end{array}$} \\
\hline & $\begin{array}{c}\text { Medi } \\
\text { a }\end{array}$ & DT & Media & DT & Media & DT & Media & DT \\
\hline 1. Verbal R. & 51,87 & 17,56 & 53,92 & 15,81 & 53,48 & 14,57 & 53,28 & 15,99 \\
\hline 2. Numérico R. & 44,43 & 17,73 & 47,92 & 16,81 & 50,32 & 17,26 & 47,61 & 17,26 \\
\hline 3. Abstracto R. & 52,27 & 22,52 & 54,83 & 22,24 & 53,09 & 21,29 & 53,75 & 22,07 \\
\hline 4. Mecánico R. & 56,91 & 15,32 & 59,29 & 14,94 & 58,78 & 14,00 & 58,55 & 14,83 \\
\hline 5. Espacial R. & 43,89 & 20,78 & 47,01 & 20,70 & 44,42 & 19,22 & 45,57 & 20,39 \\
\hline 6. Ortografía & 55,31 & 15,80 & 55,62 & 16,12 & 55,04 & 15,61 & 55,39 & 15,89 \\
\hline 7.Velocidad Pe. & 49,36 & 13,28 & 52,26 & 13,28 & 56,27 & 12,64 & 52,50 & 13,33 \\
\hline 8. Memoria & 29,99 & 11,12 & 31,59 & 10,69 & 31,81 & 9,62 & 31,23 & 10,56 \\
\hline
\end{tabular}

\footnotetext{
${ }^{1}$ La transformación se hizo de la siguiente manera: la puntuación más elevada de cada variable se elevó a 100, después se re-calcularon las demás puntuaciones siguiendo una regla de tres.
} 


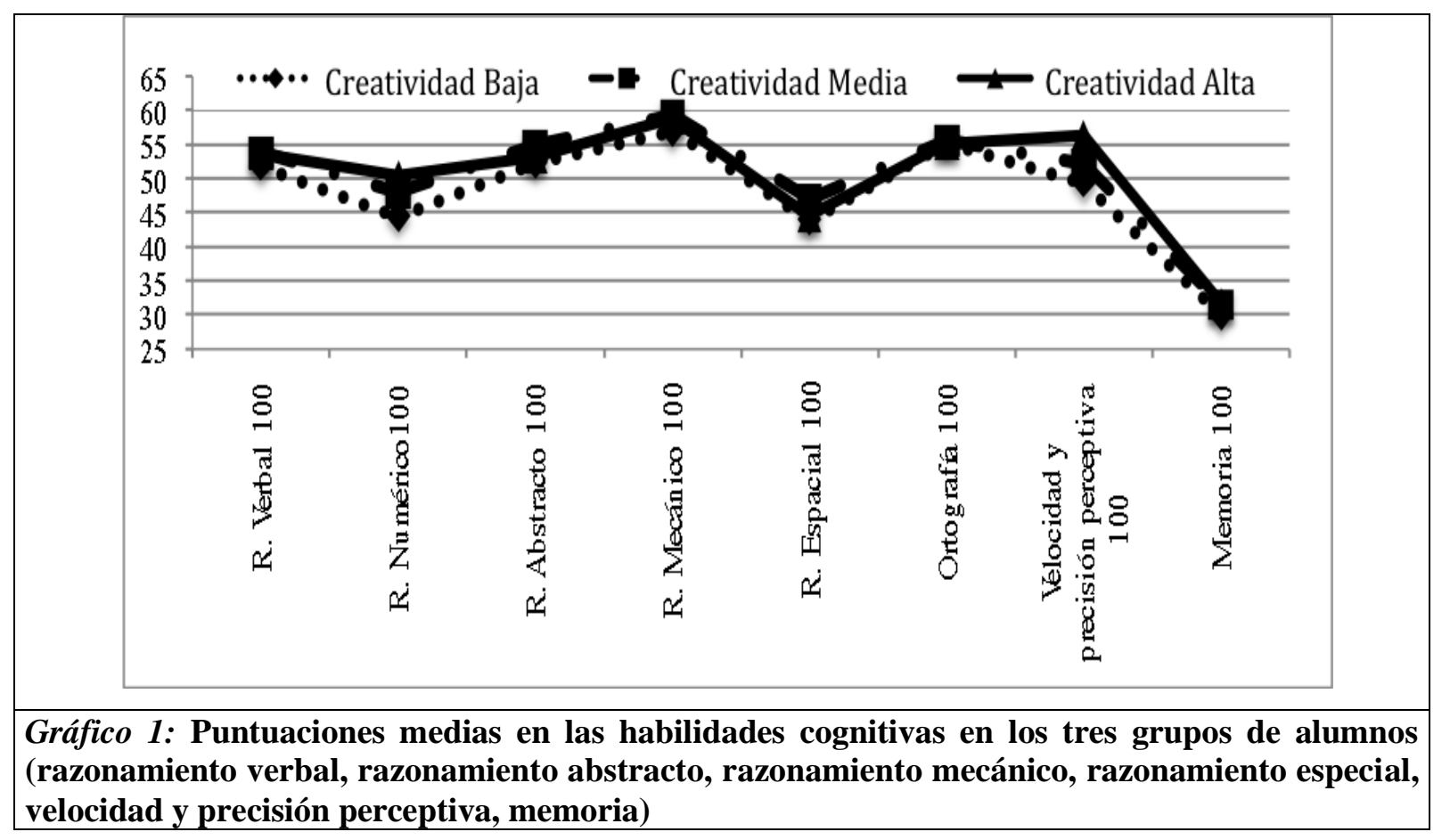

Correlaciones entre las variables y diferencias en las capacidades cognitivas según el nivel de creatividad

En la tabla 2 se muestra la correlación entre las dimensiones de creatividad y las variables del DAT-5. Las correlaciones entre la creatividad y las capacidades cognitivas oscilaron desde -,001 para razonamiento abstracto a ,167 para velocidad y precisión perceptiva. Solo hubo dos correlaciones que fueron estadísticamente significativas: razonamiento numérico $(r=, 097 ; p=, 015)$ y velocidad y precisión perceptiva $(r=, 167 ; p<$ ,001).

Cuando se analizó si existían diferencias estadísticamente significativas en inteligencia dependiendo del nivel de creatividad de los estudiantes, se encontró que existen tales diferencias sólo para el razonamiento numérico y para la velocidad y precisión perceptiva (tabla 2). El análisis de contrastes post hoc indicó que las diferencias en la velocidad y precisión perceptiva son estadísticamente significativas entre los tres grupos, mientras que la diferencia en las puntuaciones de razonamiento abstracto son estadísticamente significativas entre los alumnos con nivel de creatividad bajo y alto. 
Tabla 2: Correlaciones entre creatividad y las variables de las dimensiones cognitivas y resultados de la prueba de las diferencias de medias entre baja, media y alta creatvidad en los alumnos

\begin{tabular}{|c|c|c|}
\hline & $\begin{array}{l}\text { Correlación con } \\
\text { creatividad }\end{array}$ & ANOVAs \\
\hline 1. R. Verbal &,- 002 & $\mathrm{~F}(2,635)=, 542 ; p=, 582$ \\
\hline 2. R. Numérico &, $097 *$ & $\mathrm{~F}(2,624)=3,834 ; p=, 022^{(\mathrm{a})}$ \\
\hline 3. R. Abstracto &,- 001 & $\mathrm{~F}(2,634)=, 848, p=, 429$ \\
\hline 4. R. Mecánico & 041 & $\mathrm{~F}(2,625)=, 98 ; p=, 376$ \\
\hline 5. R. Espacial &, 018 & $\mathrm{~F}(2,623)=1,125: p=, 325$ \\
\hline 6. Ortografía &,- 006 & $\mathrm{~F}(2,633)=, 025 ; p=, 976$ \\
\hline 7. Velocidad &, $167 * *$ & $\mathrm{~F}(2,617)=11,221 ; p<, 001^{(\mathrm{b})}$ \\
\hline 8. Memoria &, 057 & $\mathrm{~F}(2,620)=, 891 ; p=, 411$ \\
\hline
\end{tabular}

El análisis de los perfiles de las capacidades cognitivas: valles y picos

Finalmente se analizó si existían diferencias en la configuración de las habilidades cognitivas según el nivel de creatividad de los estudiantes.

Con el fin de estudiar el perfil cognitivo de los participantes se utilizó una prueba de medidas repetidas. El perfil de habilidades cognitivas de cada grupo fue estudiado de forma separada. Al utilizar medidas repetidas es importante disponer de todas las variables en la misma escala, por lo tanto, las variables se transformaron de manera que la puntuación máxima fue 100 como se expuso en la sección de análisis de datos. La prueba multivariable indicó que ninguno de los tres grupos de estudiantes presenta un perfil plano, y que las diferencias entre las puntuaciones en diferentes capacidades cognitivas fueron estadísticamente significativas. Los valores para el grupo de estudiantes con bajo nivel de creatividad fueron Wilks lambda $=, 138 ; \mathrm{F}(145,7)=129,03 ; p<, 001 \eta^{2}=, 862$. Para el grupo de alumnos con un nivel de creatividad medio los resultados fueron Wilks lambda =,149; $\mathrm{F}(288,7)=235,39 ; p<, 001 ; \eta^{2}=, 851$. Y para el grupo de estudiantes con alto nivel de creatividad fueron de Wilks lambda $=, 147 ; \mathrm{F}(138,7)=114,126 ; p<, 001 ; \eta^{2}=, 853$. Con el fin de conocer donde se daban las diferencias dentro de cada perfil, se ralizó un análisis de 
contrastes post hoc mediante la comparación de los efectos principales. El nivel de significación del análisis post hoc se muestra en la tabla 3. Cuando se analizan las diferencias entre pares de variables, los tres grupos tienen resultados similares, los estudiantes de bajo nivel de creatividad difieren significativamente en 22 pares de variables, los estudiantes con un nivel de creatividad medio difieren significativamente en 23 pares de variables y los alumnos altamente creativos difieren en 21 pares de variables.

Tabla 3: Nivel de significación del análisis post hoc de las diferencias de medias entre las capacidades cognitivas en función del grupo de creatividad

\begin{tabular}{|c|c|c|c|c|}
\hline & & $\begin{array}{c}\text { Creatividad } \\
\text { Baja }(n=152)\end{array}$ & $\begin{array}{c}\text { Creatividad } \\
\text { Media } \\
(\mathrm{n}=295)\end{array}$ & $\begin{array}{l}\text { Creatividad Alta } \\
\quad(n=145)\end{array}$ \\
\hline \multirow[t]{7}{*}{ 1. R. Verbal } & 2. R. Numérico & $<, 001$ & $<, 001$ & 0,023 \\
\hline & 3. R. Abstracto & 0,802 & 0,379 & 0,781 \\
\hline & 4. R. Mecánico & $<, 001$ & $<, 001$ & $<, 001$ \\
\hline & 5. R. Espacial & $<, 001$ & $<, 001$ & $<, 001$ \\
\hline & 6. Ortografía & 0,01 & 0,073 & 0,216 \\
\hline & 7. Velocidad & 0,078 & 0,112 & 0,042 \\
\hline & 8. Memoria & $<, 001$ & $<, 001$ & $<, 001$ \\
\hline \multirow[t]{6}{*}{ 2. R. Numérico } & 3. R. Abstracto & $<, 001$ & $<, 001$ & 0,093 \\
\hline & 4. R. Mecánico & $<, 001$ & $<, 001$ & $<, 001$ \\
\hline & 5. R. Espacial & 0,738 & 0,444 & $<, 001$ \\
\hline & 6. Ortografía & $<, 001$ & $<, 001$ & 0,001 \\
\hline & 7. Velocidad & $<, 001$ & $<, 001$ & $<, 001$ \\
\hline & 8. Memoria & $<, 001$ & $<, 001$ & $<, 001$ \\
\hline \multirow[t]{5}{*}{ 3. R. Abstracto } & 4. R. Mecánico & 0,002 & $<, 001$ & 0,001 \\
\hline & 5. R. Espacial & $<, 001$ & $<, 001$ & $<, 001$ \\
\hline & 6. Ortografía & 0,101 & 0,513 & 0,256 \\
\hline & 7. Velocidad & 0,073 & 0,035 & 0,08 \\
\hline & 8. Memoria & $<, 001$ & $<, 001$ & $<, 001$ \\
\hline \multirow[t]{4}{*}{ 4. R. Mecánico } & 5. R. Espacial & $<, 001$ & $<, 001$ & $<, 001$ \\
\hline & 6. Ortografía & 0,282 & 0,001 & 0,021 \\
\hline & 7. Velocidad & $<, 001$ & $<, 001$ & 0,097 \\
\hline & 8. Memoria & $<, 001$ & $<, 001$ & 0 \\
\hline \multirow[t]{3}{*}{ 5. R. Espacial } & 6. Velocidad & $<, 001$ & $<, 001$ & $<, 001$ \\
\hline & 7. Rapidez & 0,001 & $<, 001$ & $<, 001$ \\
\hline & 8. Memoria & $<, 001$ & $<, 001$ & $<, 001$ \\
\hline \multirow[t]{2}{*}{ 6. Ortografía } & 7. Velocidad & $<, 001$ & $<, 001$ & 0,407 \\
\hline & 8. Memoria & $<, 001$ & $<, 001$ & $<, 001$ \\
\hline 7. Velocidad & 8. Memoria & $<, 001$ & $<, 001$ & $<, 001$ \\
\hline \multicolumn{2}{|c|}{$\begin{array}{l}\text { Número de pares totales que son } \\
\text { significativamente diferentes }\end{array}$} & 22 pairs & 23 pairs & 21 pairs \\
\hline
\end{tabular}




\section{Discusión y Conclusiones}

Este estudio ha tratado de arrojar algo de luz sobre la cuestión de qué hace que el pensamiento creativo ocurra. Según los datos de nuestro estudio podemos decir que la correlación entre inteligencia y creatividad es baja. Esta correlación fue estadisticamente significativa solo con las variables velocidad y precisión perceptiva y razonamiento numérico; es más nuestros resultados en general apuntan a la no existencia de diferencias estadisticamente significativas en habilidades cognitivas entre los estudiantes de alta, baja y media creatividad. Estos datos no confirman la teoría del umbral: la creatividad e inteligencia se muestran como constructos independientes, lo cual esta en acuerdo con otras investigaciones (Ferrando, Prieto, Ferrandiz y Sánchez, 2005; Kim, 2005; Naderi y Abdullah, 2010; Preckel, Holling y Wiese 2006; Runco y Albert, 1986).

Nuestra hipótesis es que los alumnos creativos no se definen por sus puntuaciones en el Cociciente Intelectual, sino por una configuración de perfiles de capacidades cognitivas. Sin embargo, los resultados de este estudio no apoyan la hipótesis de que los alumnos más creativos tengan un perfil cognitivo más plano ¿Por qué? Hay una respuesta simple: la hipótesis es errónea. Sin embargo, hay algunas limitaciones en este estudio que deberían ser tenidas en cuenta.

Entre sus limitaciones, y probablemente la mayor, es el hecho de que la creatividad se ha evaluado con una medida tradicional (TTCT, Torrance, 1974). Es discutible si el uso de procedimientos psicométricos podría identificar con precisión la creatividad, y existe la preocupación de que la creatividad sea más amplia que las cuatro dimensiones principales identificadas por Guilford (1950).

Autores como Amabile (1983) y Sternberg (Chart, Grigorenko y Sternberg, 2008; Sternberg, 2010; Sternberg, et al., 2004) proponen utilizar pruebas más holísticas, que se basan principalmente en el criterio del evaluador más que en un conjunto de parámetros rígidos. Otra limitación es que para llevar a cabo el ANOVA para medidas repetidas, las variables deben estar en la misma escala y para lograrlo las variables fueron transformadas. Por tanto, es posible que otras tareas diseñadas para que las puntuaciones estuvieran dentro del mismo rango. 
Finalmente, hemos de destacar que aun cuando la hipótesis no se pudo confirmar, este estudio muestra su mayor fortaleza en la propuesta de una nueva forma de entender la relación entre inteligencia y creatividad, ofreciendo un nuevo enfoque que puede explicar tal relación. $\mathrm{Y}$ aunque en este primer estudio la hipótesis no se comprobó, en futuras investigaciones se debería volver a poner a prueba la hipótesis utilizando otras tareas de creatividad (que pueden incluir además de tareas de pensamiento divergente, auto-informes o informes de los profesores sobre la creatividad de sus alumnos), o limitando la muestra a alumnos con inteligencia media y alta, ya que es posible que la hipótesis del perfil plano sirva para explicar las diferencias en creatividad unicamente entre los alumnos potencial cognitivo. Sabemos que los alumnos de baja inteligencia tienden a mostrar baja creatividad, pero sigue abierta la cuestión ¿por qué alumos de alta inteligencia pueden o no ser creativos?. 


\section{Agradecimientos}

El trabajo realizado ha sido posible gracias al proyecto financiado por el Ministerio de Ciencia y Tecnología (EDU2009-12925, titulado: Inteligencia Académica, Creativa y Práctica en alumnos con Superdotación y Talento) y al financiado por la Agencia de Ciencia y Tecnología de la Región de Murcia (1896/PHCS/09)

\section{Referencias}

Almeida, L. S. y Freire, T. (2003). Metodologia da Investigação em Psicologia e Educação. Braga: Psiquilíbrios.

Almeida, L., Prieto, M. D., Ferrando, M., Oliveira, E. y Ferrándiz, C. (2007). Torrance Test of Creative Thinking: the question of its construct validity. Thinking Skills and Creativity, 3, 53-58.

Amabile, T. (1983). The Social Psycholoy of Creativity: A Componential Conceptualization. Journal of Personality and Social Psychology, 45(2), 357-376.

Batey, M., y Furnham, A. (2006). Creativity, intelligence, and personality: A critical review of the scattered literature. Genetic, Social, and General Psychology Monographs, 132, $355-429$.

Bennett, G. Seashore, G. y Wesman, A. (2000). DAT-5, Test de Aptitudes Diferenciales. Madrid: TEA Ediciones.

Castelló, A. y Batlle, C. (1998). Aspectos teóricos e instrumentales en la identificación del alumno superdotado y talentoso. Propuesta de un protocolo. FAISCA, 6, 26-66.

Chart, H., Grigorenko, E. L. y Sternberg, R. J. (2008). Identification: The Aurora Battery. In J. A. Plucker y C. M. Callahan (Eds.), Critical issues and practices in gifted education (pp. 345-365). Waco, TX: Prufrock Press. 
Ferrando, M., Ferrándiz, C., Bermejo, M.R., Parra, J., Sánchez, C. y Prieto, M.D. (2007). Estructura Interna y Baremación del test de Pensamiento Creativo de Torrance. Psicothema, 19(3), 489-496.

Ferrando, M., Ferrándiz, C., Prieto, M.D., Bermejo, M.R. y Sainz, M. (2008). Creativity in Gifted \& Talented Children. The International Journal of Creativity and Problem Solving, 18(2), 35-47.

Ferrando, M., Prieto, M.D., Ferrándiz, C y Sánchez, C. (2005) Inteligencia y Creatividad. Electronic Journal of Research in Educational Psychology (7), 21-49.

Gardner, H. (1983). Frames of mind. The theory of multiples intelligences. New York: Basic Books. (Traducción castellano, Estructuras de la mente. La teoría de las Inteligencias Múltiples. México: Fondo de Cultura Económica, 1987. Última Edición 2001).

Gardner, H. (1993). Creating minds: An anatomy of creativity. New York: Basic Books. (Traducción castellano Mentes creativas: Una anatomía de la creatividad. Barcelona: Paidós, 2002).

Guilford, J. P. (1950). Creativity. The American Psychologist, 5, 444-454.

Guilford, J. P., \& Hoepfner, R. (1971). The analysis of intelligence. New York, NY: McGraw-Hill.

Hunter, S. T. y Ligon, G. S. (2008) Applying Multiple Knowledge Structures in Creative Thought effects on idea generation and problem-solving. Creativity Research Journal, 20(2) 137-154.

Kim, K. H., Cramond, B. y VanTassel-Baska, J. (2010). The relationship between creativity and intelligence. In J. C. Kaufman, \& R. J. Sternberg (Eds.), The Cambridge handbook of creativity (pp. 395-412). New York, NY: Cambridge University Press.

Kim, K. H. (2005). Can Only Iintelligence People Be Creative? The Journal of Secondary Gifted Education, 2/3, 57-66.

Mednick, S. A. (1962). The associative basis of the creative process. Psychological Review, 69, 220-232.

Mercader, M. (2012). Un Estudio de la Relación entre la Inteligencia y la Creatividad: perfil cognitivo de los alumnos de alta, media y baja creatividad. Unpublished Master The- 
sis. Universidad de Murcia.

Naderi, N. y Abdullah, A. (2010). Creativity as a predictor of intelligence among undergraduate students, Journal of American Science, 6, 86-90.

Nusbaum, E. C. y Silvia, P. J. (2011). Are intelligence and creativity really so different? Fluid intelligence, executive processes, and strategy use in divergent thinking. Intelligence, 39, 36-45.

Preckel, F. Holling, H. y Wiese, M. (2006). Intelligence and creativity in gifted and nongifted students: An investigation of threshold theory. Personality and Individual Differences, 40, 159-170.

Prieto, M. D., López, O., Ferrándiz, C. y Bermejo, R. (2003). Adaptación de la prueba figurativa del test de pensamiento creativo de Torrance en una muestra de alumnos de los primeros niveles educativos. Revista de Investigación Psicoeducativa, 21(1), 201-213.

Runco, M. A. (2007). Creativity. San Diego, CA: Academic Press.

Runco, M. A. y Albert, R. S. (1986). The threshold theory regarding creativity and intelligence: an empirical test with gifted and nongifted children. Creative Child and Adult Quarterly 11(4), 212-218.

Stemberg, R. J. The Rainbow Project Collaborators, \& University of Michigan Business School Project Collaborators (2004). Theory-Based University Admissions Testing for a New Millennium. Educational Psychologist, 59(3), 185-198.

Sternberg, R. J. (1985). Beyond IQ: A Triarchic Theory of Human Intelligence. (D. d. Brower, Trad.) New York: Cambridge University Press. (Traducción castellano, 1990. Más allá del C.I. Bilbao: DDB).

Sternberg, R. J. (2010). Assessment of gifted students for identification purposes: New techniques for a new millennium. Learning and Individual Differences, 20, 327-336.

Sternberg, R. J. y O’Hara, L. A. (1999). Creativity and intelligence. In: Sternberg, R. J., Editor, 1999. Handbook of creativity (pp. 251-272) Cambridge University Press, Cambridge: England.

Torrance, E. P. (1962). Guiding Creative Talent. Englewood Cliffs: Prentice Hall. 
Torrance, E. P. (1974). The Torrance Tests of Creative Thinking - Norms-Technical Manual Research Edition - Verbal Tests, Forms A and B - Figural Tests, Forms A and B. Princeton NJ: Personnel Press.

Weisberg, R. W. (2006). Creativity: Understanding innovation in problem solving, science, invention, and the arts. Hoboken, NJ: Wiley. 